\title{
DESIGUALDADES SOCIALES, DERECHOS Y PROTECCIÓN SOCIAL EN AMÉRICA LATINA: CONSTRUCCIONES, LUCHAS Y RESISTENCIAS
}

\author{
DESIGUALDADES SOCIAIS, DIREITOS E PROTEÇÃO SOCIAL NA AMÉRICA LATINA: CONSTRUÇÕES, LUTAS \\ E RESISTENNCIAS
}

\author{
SOCIAL DIFFERENCES, RIGHTS AND SOCIAL PROTECTION IN LATIN AMERICA: CONSTRUCTIONS, \\ FIGHTS AND RESISTANCE
}

\author{
Rodrigo Cristiano Diehl \\ Universidade de Santa Cruz do Sul \\ Estudiante de Doctorado y Magíster en Derecho de la Universidade de Santa Cruz do Sul \\ Becario Prosuc - Capes (Coordenação de Aperfeiçoamento de Pessoal de Nível) \\ Porto Alegre / RS / Brasil \\ rodrigocristianodiehl@live.com \\ (iD) Marli Marlene Moraes da Costa \\ Universidade de Santa Cruz do Sul \\ Doctora en Derecho de la Universidade Federal de Santa Catarina \\ Profesora del PPG Derecho de la Universidade de Santa Cruz do Sul \\ Santa Cruz do Sul / RS / Brasil \\ marlim@unisc.br
}

\begin{abstract}
Resumen: Este estudio tiene como objetivo analizar las desigualdades sociales y la concreción de los derechos sociales en Latinoamérica a través del proceso histórico de construcciones y luchas sociales hasta llegar a los movimientos actuales de resistencia contra el desmantelamiento constante de los sistemas de protección social. En este contexto de investigación, buscamos responder a la siguiente pregunta: ¿Qué perspectivas deben ser planteadas al analizarse las desigualdades sociales y la seguridad de los derechos sociales en América Latina, basándose en los procesos de lucha, resistencia y desmantelamiento de los sistemas de protección social? En cuanto a la metodología aplicada en el estudio, esta se divide en tres ejes: I) para lograr los objetivos inicialmente propuestos se ha utilizado la investigación exploratoria-descriptiva; II) para organizar y recopilar los materiales del estudio, se ha realizado una investigación bibliográfica cuantitativa y cualitativa; y III) para el tratamiento de los datos, se ha empleado el método del materialismo-dialéctico.
\end{abstract}

Palabras clave: América Latina; Estado; protección social.

Resumo: O objetivo com o presente estudo é analisar as desigualdades sociais e a concretização de direitos na América Latina utilizando por base todo o processo histórico de construções e lutas até os atuais movimentos de resistências frente aos constantes desmontes dos sistemas de proteção social. Diante desse contexto da pesquisa, questiona-se: quais são as perspectivas que devem ser levantadas para analisar as desigualdades sociais e a não garantia de direitos na América Latina utilizando como base os processos de lutas, resistências e desmontes dos sistemas de proteção social? A metodologia aplicada na pesquisa está dividida em três eixos: para alcançar os objetivos inicialmente propostos será utilizado a pesquisa exploratóriadescritiva; para organizar e coletar os materiais ao estudo utilizar-se-á da pesquisa bibliográfica de caráter quanti-quali e; para o tratamento desses dados o método a ser empregado será o materialismo-dialético.

Palavras-chave: América Latina; Estado; proteção social.

Abstract: The objective of the present study is to analyze social inequalities and the realization of rights in Latin America using the historical process of constructions and struggles up to the current movements of resistance against the constant dismantling of social protection systems. Given this research context, the question is: what are the perspectives that must be raised to analyze social inequalities and the non-guarantee of rights in Latin America based on the processes of struggles, resistance and dismantling of social protection systems? The methodology applied in the research is divided into three axes: to achieve the initially proposed objectives will be used exploratory-descriptive research; to organize and collect the materials for the study, it will be used the quantitative and qualitative bibliographic research; for the treatment of these data the method to be employed will be dialectical materialism.

Keywords: Latin America; state; social protection.

Para citar este artigo (ABNT NBR 6023:2018)

DIEHL, Rodrigo Cristiano; COSTA, Marli Marlene Moraes da. Desigualdades sociales, derechos y protección social en américa latina: construcciones, luchas y resistencias. Revista Thesis Juris - RTJ, São Paulo, v. 10, n. 1, p. 67-83, jan./jun. 2021. http://doi.org/10.5585/rtj.v10i1.17847. 


\section{Consideraciones iniciales}

Construir un proceso dialógico abarcando las desigualdades sociales, los derechos sociales y la protección social en los días de hoy, es decir, en la actual fase contemporánea del capitalismo marcado por el avance del conservadurismo y el dominio del capital neoliberal en los Estados latinoamericanos, no es tarea fácil. Es por eso que, pensar en los derechos sociales y en las políticas públicas implica comprender el significado y los fundamentos que los respaldan, de modo que sea posible actuar en la lucha contra las extremas desigualdades sociales, económicas y políticas.

Teniendo por base la crítica al acuerdo político que resultó de la trayectoria del fordismo y del keynesianismo en la configuración de las políticas de la era socialdemócrata, las ideas conservadoras (mantener el status quo) no solo salieron victoriosas, sino que sirvieron de fundamento para la mayoría de los países del mundo. Asimismo, los períodos conocidos como Tatcherist (en el Reino Unido) y Reaganist (en los Estados Unidos) ayudaron a difundir las ideas neoliberales, convirtiendo el Estado mínimo en un Estado hegemónico. Al mismo tiempo, durante estos períodos, el consenso social democrático de las sociedades abrió espacio para que el Consenso de Washington promoviera, según Matta (2013), una lista de contrarreformas estructurales en los servicios sociales y sus financiamientos. De acuerdo con este autor, las estrategias de contrarreforma pueden ser agrupadas en dos bloques: I) las llamadas contrarreformas de primera generación que abordan las actividades productivas y los servicios públicos esenciales a través, especialmente, de la privatización; y II) las contrarreformas de segunda generación orientadas a los sistemas de protección social.

Dado este contexto, el objetivo de este estudio es analizar las desigualdades sociales y la concretización de los derechos sociales en América Latina, teniendo por base todo el proceso histórico de construcciones y luchas sociales hasta llegar a las actuales resistencias frente al constante desmantelamiento de los sistemas de protección social. Es de importancia mencionar que, al dialogar sobre las desigualdades, los derechos sociales y los sistemas de protección social en América Latina, no podemos olvidarnos de las innumerables especificidades que existen en cada Estado. Y que, además, debemos reunir elementos cercanos que permitan la construcción de vínculos, con el fin de intercambiar experiencias y mejorar las políticas públicas de enfrentamiento a las desigualdades sociales.

Para alcanzar el objetivo del estudio, la vía metodológica utilizada fue la investigación exploratoria-descriptiva. En lo que respecta a los procedimientos, fue empleada la investigación bibliográfica cuanti-cuali, y para el análisis de los datos, fue usado el método del materialismo- 
dialéctico con el fin de permitir aproximaciones a los fenómenos naturales y sociales desde el punto de vista dialéctico; visando comprender el modo de interpretación de la realidad, materializando lo real, sus contradicciones y sus fuerzas.

Lo que nos lleva a la pregunta del estudio: ¿Qué perspectivas deben plantearse en el análisis de las desigualdades sociales y la no concretización de los derechos sociales en América Latina, basándose en los procesos de lucha, resistencia y desmantelamiento de los sistemas de protección social?

\section{Las desigualdades sociales y la protección social en américa latina: proceso de construcción, lucha y resistencia en las políticas sociales}

El proceso de deconstruir lo poco que se construyó en nombre de los dictados internacionales proviene de varios siglos atrás. Según Mattel (2013), América Latina tiene varias marcas históricas que se remontan hasta el proceso de colonización (o explotación); en donde el espacio territorial fue utilizado para la producción agrícola y el suministro de bienes primarios, sirviendo a los intereses de las metrópolis portuguesa y española. Esta lógica de colonización, que establecía una estructura productiva, social y económica orientada al exterior, ha dejado huellas que todavía perduran en el tiempo, como, la pobreza, la concentración de renta y la exclusión social.

En este campo, cobra relevancia el pensamiento de Sen (2010) que, aun haciendo distinción entre pobreza como falta de capacidad y pobreza como falta de admisión, establece que ambas están estrechamente vinculadas, ya que la admisión es un importante medio para lograr la capacitación. Sin embargo, deberíamos centrar la atención en la pobreza a partir de la falta de capacidad, dado que el aumento de la capacidad de las personas para vivir con normalidad, dentro de una normalidad social, económica y política estipulada, les permitiría aumentar su capacidad para ser más productivos y, por tanto, recibir salarios más altos. De igual manera, al considerar la necesidad de comprender la pobreza y la privación de libertades, Sen (2010) asegura que la pobreza se ha convertido en el principal problema de la sociedad capitalista y, por ende, en el foco de las políticas sociales. Motivo por lo cual, el Estado abandona la posibilidad de universalizar el acceso a los servicios básicos, y pasa a enfrentarse a la pobreza a través de la igualdad de oportunidades, de la focalización y selección de los beneficiarios, y de la transferencia de ingresos condicionada a una determinada prestación.

De los sistemas de protección social que aún permanecen de algún modo estructurados en América Latina, prevalece la identificación con la ética del workfare, es decir, la inserción en el mercado laboral como fuente de bienestar, capaz de liberar a los pobres de la pobreza y, 
consecuentemente, no generar dependencia de los individuos con relación a las prestaciones sociales. De esta manera, los sujetos pasivos y activos de los derechos sociales se hacen responsables de sí mismos y del deudor de la asistencia: de titulares del derecho pasan a ser simples beneficiarios. Sumado a esto, Stein (2017) defiende que, la moralización y la individualización de los sujetos, como principios de activación, tienen como objetivo cambiar los comportamientos y actitudes individuales, donde la cultura emprendedora se señala como la salida y la única alternativa para lograr la autonomía económica.

En contrapartida, los graves problemas y riesgos para los sistemas de protección social, constituidos en la lógica de representar una amenaza para el orden social y el trabajo, deben ser reconocidos y enfrentados, especialmente frente a las desigualdades producidas y reproducidas en el capitalismo. Otro punto de contradicción radica en el intento de contrarreformar los sistemas de protección social que, materializados en las políticas sociales, son el núcleo rígido de los Estados Democráticos de Derecho reconstruidos tras la Segunda Guerra Mundial. En este caso, la Constitución Federal brasileña de 1988, la Constitución de la Nación Argentina de 1994, la Constitución mexicana de 1917, la Constitución Política de Bolivia de 2006, por ejemplo, se colocan en el centro de las disputas político-ideológicas, a menudo, antagónicas. La política social se constituye así, como una mediación institucional político-económica que resulta, al mismo tiempo, de las contradicciones y reclamos de la lucha de clases y de la lógica de acumulación capitalista. Al presentarse como un proceso dinámico que se explica en el movimiento histórico de las sociedades, es posible reconocer la multiplicidad de perspectivas, visiones y significados de las políticas que disputan la construcción o (re)construcción de la hegemonía (SOTO; TRIPIANA, 2014).

Dentro de este proceso de construcción de las políticas sociales, Cecchini (2015) enseña que no todas están orientadas predominantemente hacia la finalidad de protección social, aunque todas tienen, en general, dimensiones de protección social. Por eso, la protección social es la parte central de la política social y esta, a su vez, es la parte esencial de los regímenes de bienestar social, que consideran no solo la acción estatal, sino también la actuación de los mercados, de las familias y de las instancias comunitarias. Por esta razón, la protección social debe materializarse a través de un conjunto de mecanismos e instrumentos que viabilicen los derechos sociales como una forma de enfrentamiento a la brecha abierta en el campo de las políticas sociales, y que tiene aumentado especialmente en los últimos años. Conjuntamente, la sociedad debe tratar de transponer la fractura histórica que resultó en las desigualdades sociales observadas en América Latina a partir de la relación entre trabajo y capital (MENDES; WÜNSCH; CAMARGO, 2011). 
Como se observa, el estudio de las políticas sociales es un fenómeno complejo, puesto que debe englobar diferentes puntos de vista, tales como: I) la importancia del éxito en los derechos sociales, culturales y económicos de los segmentos más marginados de la población, pese a que el alcance de las políticas sociales debe comprender todos los sectores de una sociedad; II) sus efectos sobre la calidad de vida de las personas como seres sociales; III) la aceptación o no de un determinado gobierno a través de la cohesión social, lo que provoca, en diferentes ocasiones, la primacía de los intereses de ciertos sectores por encima de las necesidades reales de la población; y IV) su vínculo directo con el crecimiento y desarrollo económico de un país o región (SOTO; BORREGO, 2018).

En este proceso de construcción de políticas sociales se encuentra la protección social que, en el continente latinoamericano puede entenderse desde la historia de sus modelos de desarrollo y de sus respectivos paradigmas económicos y sociales. De ahí que, Stein (2017), refiriéndose a Cecchini y Martínez (2011), identifica esta evolución con base en cuatro momentos. El primero, que comienza en el siglo XIX y termina con la crisis de 1929, estaba influenciado por el pensamiento liberal; dominado por el modelo exportador primario. En él, los indicadores sociales se caracterizan, por un lado, por la conformación de las sociedades nacionales y el sentido de pertenencia de los ciudadanos a su país y, por otro, por la caridad; en donde la atención a los problemas sociales se realiza por medio de la ayuda a los necesitados, sea a través de organizaciones de la sociedad civil o de la iglesia. Es a finales de la década de 1920, que comienza a surgir los primeros sistemas de protección social inspirados en el modelo universal (STEIN, 2017). Ya el segundo momento se extiende desde principios de la década de 1930 hasta finales de la década de 1970, y se caracteriza por el modelo sustitutivo de las importaciones, cuando los temas sociales estaban relacionados con la justicia, el orden social y la seguridad social. En este momento, tiene repercusión el papel de la sociedad civil organizada, y también de los sindicatos, en la lucha por construir modelos perfeccionados de protección social. Aquí, Stein (2017) resalta que, las limitaciones en la cobertura de los derechos sociales comprometen la perspectiva universalista de la protección social, dando prioridad a la protección contributiva de los asalariados o, en términos de protección no contributiva, actuando sólo para los grupos extremadamente vulnerables de la sociedad, dejando a una gran parte de la población fuera de estos dos sistemas. Durante este período, la gestión de las políticas sociales estaba marcada por la planificación central y predominante del financiamiento estatal, con poca participación del sector privado.

El tercer momento ocurre a finales de los años setenta y principios de los ochenta, y tiene como principales características: la crisis de la deuda pública, el déficit fiscal, las 
transformaciones del capitalismo industrial nacional en capitalismo globalizado, financiero y de servicios. En este período, las recomendaciones del Consenso de Washington y la creencia de que el mercado es el mejor instrumento para designar bienes y servicios, caracterizan el enfoque neoliberal del crecimiento externo. En cambio, la austeridad fiscal, el ajuste estructural, y los programas de estabilización económica destinados a promover el crecimiento no tuvieron en cuenta las desigualdades en la distribución de la renta (STEIN, 2017). Para el autor, la protección social en este período comienza a asumir una doble característica, la de protección contributiva y no contributiva, como forma de aliviar la pobreza extrema a través del acceso a niveles mínimos de bienestar. Cabe resaltar que, a pesar de la institucionalidad democrática y algunas acciones concretas del Estado en el área económica y social, América Latina presentó altos niveles de desigualdad y pobreza en los años ochenta y noventa.

Por fin, el cuarto momento del desarrollo histórico de la protección social identificado por Stein (2017) comienza a mediados de la década de 1990 y se extiende hasta la actualidad. Está basado en el paradigma de la competitividad sistémica, donde se hace necesario incorporar el progreso técnico al proceso productivo, con vistas a aumentar la productividad. Por consiguiente, la política social se vuelve muy importante por su contribución a la formación del capital humano, y se considera esencial para la competitividad de los países a medio plazo.

Dentro de este espacio temporal de los sistemas de protección social, Pereira (2013) contribuye al debate compilando las matrices que dan respaldo a las diversas posibilidades de estructuración y desarrollo de las medidas de protección social, que son: matriz residual, matriz socialdemócrata, y matriz socialista. 
Cuadro 01 - Cuadro comparativo de matrices teóricas e ideológicas conflictivas de protección social

\begin{tabular}{|c|c|c|c|c|c|c|c|c|}
\hline \multirow[b]{2}{*}{ Características } & \multicolumn{3}{|c|}{ MATRIZ RESIDUAL } & \multicolumn{3}{|c|}{ MATRIZ SOCIALDEMOCRATA } & \multicolumn{2}{|c|}{ MATRIZ SOCIALISTA } \\
\hline & Funcionamento & $\begin{array}{c}\text { Teoria da } \\
\text { convergência }\end{array}$ & Nova direita & $\begin{array}{l}\text { Teoria da } \\
\text { cidadania }\end{array}$ & Via média & $\begin{array}{l}\text { Administração } \\
\text { social }\end{array}$ & $\begin{array}{c}\text { Socialismo } \\
\text { democrático }\end{array}$ & $\begin{array}{c}\text { Teoria e } \\
\text { ideologia } \\
\text { marxista }\end{array}$ \\
\hline $\begin{array}{l}\text { Importância dada } \\
\text { à teoria }\end{array}$ & $\begin{array}{l}\text { Valorização de } \\
\text { teoria }\end{array}$ & $\begin{array}{c}\text { Valorização de } \\
\text { teoria }\end{array}$ & $\begin{array}{l}\text { Valorização de } \\
\text { teoria }\end{array}$ & $\begin{array}{l}\text { Valorização } \\
\text { de teoria }\end{array}$ & Pragmatismo & Pragmatismo & Pragmatismo & $\begin{array}{l}\text { Valorização de } \\
\text { teoria }\end{array}$ \\
\hline $\begin{array}{l}\text { Tipos de acordo } \\
\text { político }\end{array}$ & $\begin{array}{l}\text { Por meio do } \\
\text { consenso }\end{array}$ & $\begin{array}{l}\text { Por meio do } \\
\text { consenso }\end{array}$ & $\begin{array}{c}\text { Por meio da } \\
\text { cooptação e } \\
\text { conflito velado }\end{array}$ & $\begin{array}{l}\text { Por meio do } \\
\text { consenso }\end{array}$ & $\begin{array}{l}\text { Por meio do } \\
\text { consenso }\end{array}$ & $\begin{array}{l}\text { Por meio do } \\
\text { consenso }\end{array}$ & $\begin{array}{l}\text { Por meio do } \\
\text { consenso }\end{array}$ & $\begin{array}{l}\text { Por meio do } \\
\text { conflito }\end{array}$ \\
\hline $\begin{array}{l}\text { Tipo de proteção } \\
\text { social }\end{array}$ & $\begin{array}{l}\text { Mínima e } \\
\text { Residual }\end{array}$ & $\begin{array}{l}\text { Mínima e } \\
\text { Residual }\end{array}$ & $\begin{array}{l}\text { Mínima e } \\
\text { Residual }\end{array}$ & $\begin{array}{l}\text { Proteção } \\
\text { social ampla. } \\
\text { Pode ser } \\
\text { universal ou } \\
\text { focalizada }\end{array}$ & $\begin{array}{c}\text { Proteção social } \\
\text { ampla. Pode ser } \\
\text { universal ou } \\
\text { focalizada }\end{array}$ & $\begin{array}{c}\text { Proteção social } \\
\text { ampla. Pode ser } \\
\text { universal ou } \\
\text { focalizada }\end{array}$ & $\begin{array}{c}\text { Proteção social } \\
\text { universal }\end{array}$ & $\begin{array}{c}\text { Proteção social } \\
\text { universal }\end{array}$ \\
\hline $\begin{array}{l}\text { Principais } \\
\text { instancias } \\
\text { provedoras }\end{array}$ & Estado e Mercado & $\begin{array}{l}\text { Estado e } \\
\text { Mercado }\end{array}$ & $\begin{array}{c}\text { Mercado e } \\
\text { outras } \\
\text { instâncias } \\
\text { privadas não } \\
\text { mercantis } \\
\text { (família, ONG) }\end{array}$ & Estado & $\begin{array}{c}\text { Pluralismo de } \\
\text { Bem- } \\
\text { Estar (Estado, } \\
\text { mercado e } \\
\text { instâncias privadas } \\
\text { não mercantis) }\end{array}$ & Estado & Estado & Estado \\
\hline Status de direito & $\begin{array}{l}\text { Proteção social } \\
\text { não é direito }\end{array}$ & $\begin{array}{l}\text { Proteção social } \\
\text { não é direito }\end{array}$ & $\begin{array}{l}\text { Proteção social } \\
\text { não é direito }\end{array}$ & $\begin{array}{l}\text { Proteção } \\
\text { social é } \\
\text { direito }\end{array}$ & $\begin{array}{c}\text { Proteção social é } \\
\text { direito }\end{array}$ & $\begin{array}{c}\text { Proteção social é } \\
\text { direito }\end{array}$ & $\begin{array}{c}\text { Proteção social } \\
\text { é direito }\end{array}$ & $\begin{array}{c}\text { Proteção social é } \\
\text { e não é direito } \\
\text { (contraditória). }\end{array}$ \\
\hline $\begin{array}{l}\text { Objetivo primeiro } \\
\text { da proteção social }\end{array}$ & $\begin{array}{l}\text { Preservação do } \\
\text { capitalismo }\end{array}$ & $\begin{array}{l}\text { Preservação do } \\
\text { capitalismo }\end{array}$ & $\begin{array}{l}\text { Preservação do } \\
\text { capitalismo }\end{array}$ & $\begin{array}{c}\text { Preservação } \\
\text { do } \\
\text { capitalismo }\end{array}$ & $\begin{array}{l}\text { Preservação do } \\
\text { capitalismo }\end{array}$ & $\begin{array}{l}\text { Preservação do } \\
\text { capitalismo }\end{array}$ & $\begin{array}{l}\text { Superação do } \\
\text { capitalismo e } \\
\text { emancipação } \\
\text { humana }\end{array}$ & $\begin{array}{c}\text { Pode contribuir } \\
\text { para a superação } \\
\text { do capitalismo e } \\
\text { emancipação } \\
\text { humana E para a } \\
\text { conservação do } \\
\text { capitalismo e da } \\
\text { alienação } \\
\text { humana. }\end{array}$ \\
\hline
\end{tabular}

Fonte: Pereira, 2013. 
El autor explica que, en la Matriz Residual la protección social está permitida siempre que sea puntual, eventual, condicionada, focalizada y mínima. Su principal objetivo en este momento es promover la cohesión, el orden y la armonía social que son imprescindibles para el pleno funcionamiento de la sociedad capitalista. En este entorno, las instituciones privadas de mercado y de no mercado (por ejemplo, familias, asociaciones y organizaciones no gubernamentales) desempeñan un papel de destaque en la provisión de la protección social (PEREIRA, 2013). Por otra parte, en la Matriz Socialdemócrata, aunque se comparta la misma idea de protección social como elemento de cohesión de la sociedad capitalista, se introduce un nuevo elemento: su reconocimiento como un derecho a ser garantizado. No obstante, Pereira (2013) advierte que la universalidad no se presenta como un eje central y unánime de acción, mismo comprendiéndose su importancia estratégica para prevenir las crisis y resolver los problemas sociales. En cuanto a la Matriz Socialista, esta entiende la protección social de carácter público, gratuito y universal, como posibilidad de satisfacer las necesidades humanas y solucionar los males sociales causados y perpetuados por el capitalismo (PEREIRA, 2013). De esta forma, además de enmarcarse como un derecho básico de todas las personas, la matriz socialista de la protección social garantiza el desarrollo con calidad de las sociedades, ya que mitiga el daño producido por el capitalismo.

Ante el escenario de expansión selectiva de las políticas de protección social, a diferencia de lo previsto en varios textos constitucionales latinoamericanos, incluido el brasileño, se verifica que no se constituye como una estrategia de universalización de los derechos sociales. Lo que realmente sucede, de acuerdo con Barreto (2016), es que el Estado, imbuido de anhelos neoliberales, intensifica el asistencialismo, la mercantilización y la privatización de la protección social, es decir, expande la asistencia social, mercantiliza la salud y restringe el acceso a la previdencia social a través de contrarreformas, que favorecen la seguridad social privada complementaria: los famosos fondos de pensiones.

En este punto de desarrollo del capital de orientación neoliberal, no podemos olvidarnos que la inserción de países clasificados como periféricos dentro del capitalismo también refleja la división internacional del trabajo, que lleva sus marcas históricas de persistencia en su formación y desarrollo. Según Iamamoto (2015), el desarrollo de estas nuevas condiciones histórico-sociales metamorfosea la cuestión social que es inherente al proceso de acumulación capitalista, espesándola con nuevas determinaciones y relaciones sociales históricamente producidas, e impone el desafío de dilucidar su significado social en el presente. 
se às instituições financeiras (bancos, companhias de seguros, fundos de pensão, sociedades financeiras de investimentos coletivos e fundos mútuos), que passam a comandar o conjunto da acumulação, configurando um modo específico de dominação social e política do capitalismo, com o suporte dos Estados Nacionais (IAMAMOTO, 2015, p. 108).

Entre los posibles mecanismos de resistencia, mantenimiento y expansión de los sistemas de protección social en América Latina, la problematización en tiempos de crisis es una de las formas posibles de hacerlo. Por lo tanto, esta problematización, sobre todo en tiempos de crisis, puede servir para reducir el desempleo y hacer frente a la precariedad laboral. Esta etapa es extremamente importante, ya que su discusión implica reestructurar la base productiva, devaluar la fuerza laboral y redirigir los mecanismos de regulación estatal (MOTA, 2008). Por lo tanto, el análisis de los sistemas de protección social no puede disociarse de otros elementos, como, el social, el político y el económico. Mucho menos, de los fundamentos de la lucha de clases institucionalizada (a través del reconocimiento de grupos de capital y de trabajo) y de la función de controle del Estado para que esta lucha no afecte directamente el principio de la acumulación de plusvalía. En este contexto, la protección social aparece como un objetivo móvil de acuerdo con Valle (2012), ya que, por un lado, existe la lógica de la acumulación de quienes poseen el capital y, por el otro se encuentra la lógica de la distribución.

La protección social -clasificada como determinante en la regulación de las relaciones económicas y sociales de los individuos en el sistema de producción keynesiano-fordista- se expande a través de la seguridad social y se materializa a través de políticas sociales, y su fundamento se encuentra en el núcleo central de los Estados sociales después de la Segunda Guerra Mundial. Los derechos previstos en ella, ya sean basados en el modelo bismarckiano ${ }^{1}$ (alemán) o el modelo beveridgiano ${ }^{2}$ (inglés), tienen como parámetro las relaciones laborales y la garantía de beneficios básicos para quienes perdieron su capacidad de trabajo, ya sea de forma momentánea o permanente (BOSCHETTI, 2009). Uno de los desafíos de este escenario inicial fue la construcción de la articulación entre el problema social y la política de protección social, para que juntos pudieran comprender el contexto sociopolítico de las vulnerabilidades y de los riesgos sociales ${ }^{3}$. Lo relevante de este proceso de reestructuración de la protección social es el hecho de que, en el siglo pasado, según Fernandes (2007), la humanidad fue responsable de un

\footnotetext{
${ }^{1}$ El modelo bismarckiano, basado en el proyecto alemán de 1883 de Otto von Bismarck, puede entenderse como la génesis de la protección social conferida por el Estado. Inicialmente surgió como una forma de seguro de salud, evolucionando para incluir también seguro contra accidentes laborales, discapacidad y vejez. La financiación en este modelo se basa en el contrato y el principio de contribución adoptando la forma tripartita: empleado, empleador y Estado.

${ }^{2}$ El modelo beveridgiano, basado en el plan inglés de Lord Beveridge de 1946, comprende que la seguridad social debe proporcionarse desde la cuna hasta la tumba. Sus objetivos eran la unificación del seguro social existente, el establecimiento de la universalidad de la cobertura (todos los ciudadanos y no solo los trabajadores, por lo tanto independientes de la contribución), la protección igualitaria y la triple forma de costos (predominantemente financiación estatal

${ }^{3}$ [...] manifestações de desproteções sociais, como expressões da questão social, requerem a primazia do Estado na provisão da proteção social [...]. Entretanto, não basta reconhecer ou identificar, em um território de ação, situações de pobreza, de fragilidades nas relações familiares, de abandonos, de situações de rua, de desemprego, enfim, de violações de direitos (embora isto seja fundamental); é preciso que se compreendam as circunstâncias em que os sujeitos adentram nas zonas de vulnerabilidades sociais. A ampliação desta compreensão se dá por meio da reflexão crítica e dos espaços compartilhados para que isto ocorra [...] - pois somente assim é possível construir estratégias para a superação dessas vulnerabilidades engendradas nesta sociedade capitalista (FERNANDES, 2016, p. 232).
} 
excelente avance científico y tecnológico en varias áreas, lo que proporcionó impactos positivos en la calidad de vida de los ciudadanos. Por el contrario, en América Latina, este progreso fue extendido de forma lenta y extremadamente desigual.

Bajo este manto de contradicciones, Valle (2012) establece tres aspectos centrales: el primero hace referencia al desarrollo de sistemas de protección social en América Latina, que tiene como característica común el principio de la subsidiariedad que hasta entonces orientaba su acción y solo "autorizaba" al Estado a intervenir en las cuestiones sociales cuando las instituciones más cercanas al ciudadano, por ejemplo, la iglesia y otras asociaciones religiosas fallaran. El segundo señala que, desde el comienzo de la historia de la protección social, esta se ha segmentado en varios sistemas, que en última instancia privilegiaron y garantizaron el acceso, en particular, a los empleados que estaban vinculados a dicho sistema. Para poder ingresar en el sistema, se establecieron niveles de servicios basados en el estatus profesional y, además, se desarrollaron mecanismos de representación. De esta forma, la prestación de servicios relacionados con el bienestar individual y social se convirtió en un sistema corporativo (VALLE, 2012). Ya el tercer aspecto, relacionado con los sistemas de protección social en el contexto latinoamericano, tenía como objetivo principal proteger al proveedor de la familia (generalmente el esposo/padre), quedándose los demás miembros del núcleo familiar sin protección por parte del Estado.

Actualmente, la protección social, materializada en la seguridad social y en políticas sociales, puede considerarse como el elemento central del bienestar, constituyéndose en el mecanismo legítimo de reconocimiento y de enfrentamiento de las desigualdades sociales en América Latina, generadas por patrones de disparidad en las relaciones de producción, acumulación y concentración de riqueza socialmente producida (MENDES; WÜNSCH; SILVA, 2014). Lo que demuestra la importancia de comprender la relación existente entre la sociedad, el Estado y las clases sociales en las diversas fases de interlocución. De otro modo, Cortés y Flores (2014) definen la protección social a partir de tres dimensiones e indicadores principales: la primera sería la cobertura horizontal, es decir, la proporción de la población que de alguna manera está cubierta por la seguridad social; la segunda sería la cobertura vertical, esto es, los beneficios de un derecho efectivo y ofrecido a los afiliados (en el sistema de beneficiarios); y la tercera sería la protección social financiada, más bien, aquella medida de manera que los gastos no amplíen la desigualdad de ingresos.

Con una visión destinada a garantizar los derechos previstos en la legislación, Cecchini (2015) reconoce la protección social como una política fundamental que contribuye al desarrollo de los derechos económicos y sociales de la población, reconocidos en una maraña 
de instrumentos jurídicos nacionales e internacionales, como la Declaración Universal de los Derechos Humanos (ONU - 19484) y la Declaración Americana de Derechos y Deberes del Hombre (OEA - 19485 $)$. Estos instrumentos normativos internacionales son un ejemplo del reconocimiento de los derechos a la seguridad social, al trabajo y a la protección de las personas en niveles apropiados. Como elemento presente en el desarrollo social, la protección social debe entenderse dentro de un contexto que hace frente a la deuda social heredada del capitalismo, y que debe ser trascendida con un enfoque de totalidad capaz de captar la diferenciación y superarla para lograr la justicia social. En este contexto, el proyecto societario socialista no se reduce a la protección de los individuos, sino que también los coloca como protagonistas del desarrollo social, con el objetivo de expandir su potencial (COUTO, et al., 2018).

Sumado a esto, Mendes y Wünsch (2011) señalan que los cambios continuos que envuelven la esfera del trabajo en la sociedad actual, y que tienen repercusiones directas en la protección social, también están relacionados con cambios en el papel y en la orientación del Estado, sobre todo en el inicio de la década de 1980 y en 1990 con el adviento del paradigma neoliberal. Este, brinda la oportunidad de revelar el desajuste en la tríada capital-trabajo, Estado y protección social, liderado por la urgencia de crear nuevas formas de producción. La concesión de este desajuste en la tríada es lo que permite un análisis amplio de los fenómenos allí involucrados, bien como la visualización de las dos consecuencias más graves de las políticas neoliberales en América Latina: la ampliación de las desigualdades sociales y la ruptura del aparato industrial nacional (SALAMA, 1995). Estos efectos negativos ${ }^{6}$, generados en gran medida por los gobiernos neoliberales, pueden presentarse como el claro resultado del fracaso del propio Estado. Por un lado, está la sobreexplotación del capital y por otro la lucha infructuosa contra las desigualdades sociales. El paso inicial para esta problematización es discutir los principales modelos de materialización de la protección social en América Latina, teniendo en cuenta el centro de acción, los momentos históricos enumerados anteriormente y los modelos de protección social abordados por Fleury (1994), que son: la asistencia social, el seguro social y la seguridad social.

El primer modelo tiene su núcleo formado en la Asistencia Social y emerge en contextos socioeconómicos en los cuales el mercado es el agente controlador de las demandas sociales, y depende de cada individuo perseguir sus intereses individuales, como la adquisición de bienes

\footnotetext{
${ }^{4}$ La Declaración fue proclamada por la Asamblea General de las Naciones Unidas en París el 10 de diciembre de 1948, por la Resolución 217 A (III) de la Asamblea General como un estándar común que deben alcanzar todos los pueblos y naciones y por primera vez estableciendo protección universal de los derechos humanos. ${ }^{5}$ La Declaración es una declaración internacional adoptada en 1948 en la IX Conferencia Internacional Americana celebrada en Bogotá, la misma conferencia en la que se creó la Organización de Estados Americanos (OEA).

${ }^{6}$ Salama (1995) trae la posibilidad de efectos positivos del neoliberalismo, sin embargo, advierte que aparentemente fueron construidos, frágiles y con un alto costo social, lo que hace del neoliberalismo una experiencia débil.
} 
y servicios, que conducen a una ciudadanía invertida. En la Asistencia Social los valores predominantes son la libertad y el individualismo, objetivando la igualdad de oportunidades (FLEURY, 1994). El segundo modelo, llamado Seguro Social, tiene como elemento central la posibilidad de cubrir ciertos grupos ocupacionales a través de una relación contractual, es decir, una ciudadanía regulada. La única característica distintiva del seguro privado es el organismo que lo sanciona, en este caso el Estado. El servicio a los trabajadores se realiza mediante contribuciones previas, lo que no elimina una fuerte burocracia que busca la lealtad de los beneficiarios (FLEURY, 1994). El tercer y último modelo tiene como elemento constitutivo de la protección social la Seguridad Social en la cual el Estado, a través de un conjunto de políticas públicas, gubernamentales y unificadas, tiene como objetivo garantizar las condiciones básicas de la ciudadanía y el ideal de justicia social, incluidos los ingresos, bienes y servicios. Este sistema, según Fleury (1994), permite la redistribución de la riqueza producida socialmente y, por lo tanto, la corrección de las desigualdades sociales extremas, al mismo tiempo que vincula la ciudadanía universal a los pueblos latinoamericanos.

En este sentido, el término política pública se utiliza en este estudio con base en el siguiente concepto: las políticas públicas son respuestas del poder público a los problemas políticos. En otras palabras, las políticas designan iniciativas estatales (gobiernos y otras autoridades públicas) para satisfacer las demandas sociales relacionadas con problemas comunes a la población, que se ejecutan directamente por agencias públicas o se delegan a organizaciones civiles o privadas (SCHMIDT, 2008).

Como forma de representar los tres modelos del sistema de protección social (asistencia, seguro y seguridad), se muestra una tabla, adaptada a la creada por Fleury (1994), que sintetiza y sistematiza las ideas y modalidades principales de las definiciones dentro de estos modelos. 
Cuadro 02 - Modelos de protección social (asistencia, seguro y seguridad)

\begin{tabular}{|c|c|c|c|}
\hline MODALIDADES & ASISTENCIA & SEGURO & SEGURIDAD \\
\hline Denominaciones & Residual & Meritocrático & Institucional \\
\hline Ideología & Liberal & Corporativa & Socialdemocracia \\
\hline Principio & Caridad & Solidaridad & Justicia \\
\hline Efecto & Discriminación & Manutención & Redistribución \\
\hline Status & Descualificación & Privilegio & Derecho \\
\hline Actuaria & Fundos & Acumulación & Desglose \\
\hline Cobertura & Objetivos & Ocupacional & Universal \\
\hline Beneficios & Bienes/Servicios & Proporción Salarial & Mínimo vital \\
\hline Acceso & Tests medios & Filiación & Necesidad \\
\hline Administración & Filantrópico & Corporativo & Público \\
\hline Organización & Local & Fragmentada & Central \\
\hline Referencia & Poor Laws & Bismarck & Beveridge \\
\hline Ciudadanía & Invertida & Regulada & Universal \\
\hline
\end{tabular}

Fuente: FLEURY, 1994, p. 108.

El modelo ideal de protección social se centra en la idea de seguridad y ciudadanía universal, especialmente dadas las peculiaridades de los Estados latinoamericanos en el capitalismo periférico. Sin embargo, en varios países de la región, la política social se ha desarrollado no por la presencia del Estado de bienestar (Welfare State ${ }^{8}$ ), sino por la existencia, en su proceso de construcción, de un carácter asistencialista y clientelista, eliminando así su universalización y el reconocimiento de los derechos de la ciudadanía (MARCOSIN; SANTOS, 2010). De ahí se deriva la necesidad de estructurar la protección social en función de una visión sistémica, donde es posible encontrar los dos pilares básicos de los sistemas: el pilar no contributivo (conocido tradicionalmente a través de la acción de la asistencia social, que puede incluir medidas universales o focalizadas) y el pilar contributivo, que puede encontrar la previdencia social.

La problematización de la protección social en tiempos de crisis de capital y neoliberalismo no debe resumirse simplemente a la discusión entre crisis económica y crisis de protección social, sino que debe ser historiada, politizada y traducida, entendiéndola en un mundo donde las relaciones laborales sufren constantes cambios, y son transformadas a partir de la retirada de los derechos sociales. Dentro de este contexto, los sistemas de protección

\footnotetext{
${ }^{7}$ Poor Relief Act (la Ley de Protección de los Pobres) fue instituida en 1601 en Inglaterra y permitió a los jueces de la Comarca del condado que todos los ocupantes y usuarios de la tierra pagasen un impuesto de caridad. La cantidad recaudada se centró en las parroquias (que proporcionaron ayuda directa a los necesitados) y administrada por inspectores nombrados por los jueces.

${ }^{8}$ El Welfare State o Estado del Bienestar es un modelo de organización política y económica que coloca al Estado como un agente de promoción social y un organizador de la economía.
} 
social, constituidos en la relación entre Estado y sociedad, son determinados actualmente por un conjunto de necesidades que nascen con la producción del capital, pero no finalizan ahí. (MOTA, 2008).

En este contexto, el reconocimiento de los elementos presentes en los procesos de afirmación y expansión de los sistemas de protección social en América Latina, así como aquellos destinados a su reducción, desmantelamiento o descaracterización a través de contrarreformas, son esenciales para reducir las desigualdades sociales y luchar contra la desprotección social. La clave está en analizar la protección social como la "base" y no como la "cúspide" a alcanzar para garantizar los derechos sociales. Pese a esto, la construcción de la garantía de los derechos sociales se ve amenazada por contrarreformas estructurales impuestas por el capital neoliberal.

\section{Consideraciones finales}

El estudio sobre desigualdades sociales, derechos y protección social en los Estados latinoamericanos se basa en la necesidad de problematizar teorías eminentemente decoloniales que entiendan y tengan en cuenta las especificidades y la diversidad de las naciones. Bajo este nuevo contexto de propuesta, se puede decir que las sociedades latinoamericanas deben luchar contra los discursos y las acciones, especialmente las de cuño neoliberal, que tienen como objetivo reducir o exterminar a los sistemas de protección social y alejar la garantía de los derechos. En este contexto, es esencial percibir la explotación y la dominación como una forma de conocer la realidad, ya que el capital no puede revelarse al mundo de los fenómenos a partir de estas acciones. El capital mantiene entre sus promesas la de promover un mundo con hombres y mujeres libres e iguales que, incluso cuando sus derechos son violados por el propio sistema del capital, deben reconstruirse. Existe, por lo tanto, la creación de un mundo imaginario (ficción) que cubre y distorsiona la esencia de su ser (mundo real), donde el capital necesita presentarse de manera distorsionada, para no revelar a primera vista lo que es de hecho. Este fenómeno se llama fetichización del capital, que termina creando un mundo encantado: el capital como una fábula.

Así, en virtud de representar una de las principales fuentes de provisión de bienestar social en la sociedad latinoamericana, los sistemas públicos de protección social son contradictoriamente el objetivo de las políticas neoliberales ya conocidas, pero ahora travestidas de otros fenómenos (neoconservadurismo, por ejemplo), que promueven su desmantelamiento y su descaracterización; lo que implica directamente tanto en la 
concretización de los derechos como en la expansión de las vulnerabilidades y de las desigualdades.

La construcción de un proceso dialógico a cerca de las desigualdades sociales, la pobreza extrema y los sistemas de protección social en América Latina constituye un desafío, especialmente en la fase actual de las sociedades donde el conservadurismo, una vez más, dicta las reglas, ahora bajo el mando del neoliberalismo. Cuando se trata de la realidad latinoamericana, y la necesidad de comprender los fenómenos producidos y reproducidos en este continente, está hablándose de una región que tiene un gasto público medio de menos del $15 \%$ del PIB, mientras que las tasas de pobreza e indigencia son altas: el $28.2 \%$ y el $11.3 \%$, respectivamente (CEPAL, 2019).

Bajo este escenario, el presente estudio tuvo como objetivo responder a este problema: ¿Qué perspectivas deben ser planteadas al analizarse las desigualdades sociales y la garantía de los derechos en América Latina, en función de los procesos de lucha, resistencia y desmantelamiento de los sistemas de protección social? Contradictoriamente en su lugar, las ideologías, los valores y las prácticas que se oponen a la garantía de los derechos ganan terreno, imponiendo límites en la realización de políticas públicas de beneficio para todos, sobre la base de una nueva administración de escasos recursos públicos.

Como perspectivas para la comprensión de las desigualdades sociales y la garantía de los derechos en América Latina, es necesario verificar el intento de "mercantilizar" la propia vida en la sociedad, usurpando los derechos de la ciudadanía y, por lo tanto, disminuyendo el papel y la participación del Estado en la protección social. En este punto, se afirma que la realidad, los hechos y los eventos necesitan ser desenmascarados, de modo que haya un largo camino de construcción entre la apariencia y la esencia, entre la parte y la totalidad, entre lo singular y lo universal.

\section{Referencias}

BARRETO, L. M. P. A (ultra)precarização do trabalho como condição de acesso à Previdência Social. Revista Ser Social, Brasília, v. 18, n. 39, p. 689-704, jul./dez. 2016. DOI: https://doi.org/10.26512/ser_social.v18i39.14640

BOSCHETTI, I. Seguridade social na América Latina. In: BOSCHETTI, I et al. Política social no capitalismo: tendências contemporâneas. 2. ed. São Paulo: Cortez, 2009. CECCHINI, S. et al. Derechos y ciclo de vida: reordenando los instrumentos de protección social. In: CECCHINI, S. et. al. (Orgs.). Instrumentos de protección social: caminos latinoamericanos hacia la universalización. Santiago: Cepal, 2015, p. 25-48. 
CECCHINI, S.; MARTÍNEZ, R. Protección social inclusiva en América Latina. Una mirada integral, un enfoque de derechos. Santiago de Chile: Naciones Unidas, 2011.

CEPAL. Comissão Econômica para América Latina e Carine. Panorama social de América Latina 2018. Santiago do Chile: Cepal, 2019. Disponible en: https://www.cepal.org/ptbr/publicaciones/44412-panorama-social-america-latina-2018-documento-informativo. Aceso en 15 MAY 21.

CORTÉS, C. L.; FLORES, C. S. El Sistema de Pensiones de Chile en la Encrucijada: reformando la Gran Reforma de 2008. Revista Textos \& Contextos, Porto Alegre, v. 13, n. 01, p. 113-127, jan./jun. 2014. DOI: https://doi.org/10.15448/1677-9509.2014.1.17981

COUTO, B. R.; RIZZOTTI, M. L. A. Proteção Social no Brasil: uma análise a partir da dimensão política. In: CONSERVA, M. de S. (Org.). Multiterritoriedades e os desafios da proteção social no Brasil e na Europa. 2. ed. João Pessoa: União Editora, 2018, p. 114-132.

FERNANDES, R. M. C. Desigualdade social: retomando a reflexão social. Revista Opinio, Canoas, n. 18, p. 143-152, jan./jun. 2007. Disponible en: https://www.ulbra.br/upload/5193441101ca85c9d1e6a5e03e71b9da.pdf. Aceso en 15 MAY 21.

FERNANDES, R. M. C. Questão social. In: FERNANDES, R. M. C.; HELLMANN, A. (Orgs.). Dicionário crítico: política de assistência social no Brasil. Porto Alegre: Ed. UFRGS, 2016, p. 230-233.

FLEURY, S. Estado sem cidadãos: seguridade social na América Latina. Rio de Janeiro: Fiocruz, 1994.

IAMAMOTO, M. V. Serviço social em tempo de capital fetiche: capital financeiro, trabalho e questão social. 9. ed. São Paulo: Cortez, 2015.

MARCOSIN, C.; SANTOS, C. A acumulação capitalista e os direitos do trabalho: contradição histórica que preside a seguridade social. In: BEHRING, E. R.; ALMEIDA, M. H. T. (Orgs.). Trabalho e seguridade social: percursos e dilemas. 2. ed. São Paulo: Cortez, 2010, p. 175-192.

MATTA, J. J. da. Dos Institutos de Aposentadoria e Pensões ao Seguro Complementar: trabalho e previdência no Brasil. Revista Em Pauta, Rio de Janeiro, n. 32, v. 11, p. 173-196, $2^{\text {o }}$ sem. 2013. DOI: https://doi.org/10.12957/rep.2013.10161

MATTEL, L. Políticas sociais de enfrentamento da pobreza na América Latina: uma análise comparada entre Brasil e Argentina. Revista Katálysis, Florianópolis, v. 18, n. 2, p. 222-230, jul./dez. 2013. DOI: https://doi.org/10.1590/1414-49802015180200008

MENDES, J. M. R.; WÜNSCH, D. S. Saúde do trabalhador e proteção social: as repercussões da precarização do trabalho no capitalismo contemporâneo. Revista Estudos do Trabalho, São Paulo, v. 5, p. 1-13, 2011. Disponible en: www.estudosdotrabalho.org/10revistaRET9.pdf Aceso en 15 MAY 21. 
MENDES, J. M. R.; WUNSCH, D. S.; SILVA, L. A. M. A seguridade social: percursos e desafios no âmbito da proteção social. In: MENDES, J. M. R.; MANDALOZZO, S.; SILVA, L. A. M. (Orgs.). Trabalho e proteção social. Ponta Grossa: Estudio Texto, 2014, p. 11-30.

MOTA, A. E.; MARANHÃO, C. H.; SITCOVSKY, M. As tendências da política de Assistência Social, o SUAS e a formação profissional. In: MOTA, Ana Elizabete (Org.). O Mito da assistência social: ensaios sobre Estado, política e sociedade. 2. ed. São Paulo: Cortez, 2008.

OFFE, C. Un diseño no productivista para las políticas sociales. In: BARBEITO, R.; VUOLO, R. (Orgs.). Contra la exclusión: la propuesta del ingreso ciudadano. Buenos Aires: Miño Dávila Ed., 1997, p. 83-108.

PEREIRA, P. A. P. Proteção social contemporânea: cui prodest? Revista Serviço Social e Sociedade, São Paulo, n. 116, p. 636-651, out./dez. 2013. DOI:

https://doi.org/10.1590/S0101-66282013000400004

SALAMA, P. Para uma nova compreensão da crise. In: SADER, E.; GENTILI, P. (Orgs). Pós-neoliberalismo: as políticas sociais e o Estado democrático. São Paulo: Paz e Terra, 1995, p. 51-53.

SCHMIDT, J. P. Para entender as políticas públicas: aspectos conceituais e metodológicos. In: REIS, J. R.; LEAL, R. G. (Orgs.). Direitos sociais e políticas públicas: desafios contemporâneos. Santa Cruz do Sul: Edunisc, 2008.

SEN, A. Desenvolvimento como liberdade. São Paulo: Companhia das Letras, 2010.

SOTO, O. P.; BORREGO, A. E. Protección y política social. In: COUTO, B. R. et al. (Orgs.). Análise dos sistemas de proteção social na América Latina: Brasil, Chile e Cuba. Porto Alegre: EDIPUCRS, 2018, p. 73-84.

SOTO, S. G. F.; TRIPIANA, J. D. El desarrollo del capitalismo y la nueva configuración de la protección social. La Asignación Universal por Hijo en Argentina. Revista Textos \& Contextos, Porto Alegre, v. 13, n. 2, p. 274-290, jul./dez. 2014. DOI:

https://doi.org/10.15448/1677-9509.2014.2.17977

STEIN, R. H. La protección social en América Latina y la particularidad de la asistencia social. Revista Ser Social, Brasília, v. 19, n. 40, p. 49-68, jan./jun. 2017. DOI: https://doi.org/10.26512/ser_social.v19i40.14671

VALLE, A. H. Protección social, acción estatal y estructura de riesgos sociales. Revista Texto \& Contextos, Porto Alegre, v. 11, n. 1, p. 52-64, jan./jul. 2012. Disponible en: https://revistaseletronicas.pucrs.br/ojs/index.php/fass/article/view/9235/8053. Aceso en 15 MAY 21. 